\title{
HOW HIGH A DENSITY IS FOR PHOTOIONIZATION IN AGN?
}

\author{
Wei Zheng \\ University College London \\ Department of Physics and Astronomy \\ WC1E 6BT, England
}

\begin{abstract}
At a density of $10^{11} \mathrm{~cm}^{-3}$ or higher, the column emissivity of many lines, such as hydrogen Balmer lines, may not continue to increase with density because the strong radiative transfer results in a "skin effect" near the illuminated front. The $L y \alpha$ to Balmer line ratio is larger than at lower density.
\end{abstract}

It has been suggested (Peterson 1988) that the density of broad emission line region in AGN may be significantly greater than the "standard value", $10^{9} \mathrm{~cm}^{-3}$, (Ferland, and Netzer 1984) because of the observed short time scale of line variation. As shown in the photoionization calculation at higher density (Zheng, and Puetter 1988), the opacity of major lines can be so large that their emissivity decreases at a short distance into the cloud. This radiative transfer affects the line ratio. For example, a reasonable level of $\mathrm{C} \mathrm{III]} \mathrm{intensity} \mathrm{may} \mathrm{be} \mathrm{obtained} \mathrm{if}$ the cloud is thick enough since the $\mathrm{C} \mathrm{III]} \mathrm{emission} \mathrm{extends} \mathrm{into} \mathrm{the} \mathrm{deep} \mathrm{region.}$

The Balmer emission is significantly affected because a large portion of it originates in the deep zone. The population $n_{2}$ increases with density faster than $\mathrm{n}_{1}$ and results in relatively larger Balmer opacity. As shown in Figure 1, the total emissivity does not increase with ionizing flux at higher density. Each calculation was made for cloud of sufficient large column mass, at the order of $10^{23} \mathrm{~cm}^{-2}$. The Balmer column emissivity remains on the order of $10^{8} \mathrm{ergs} / \mathrm{s} / \mathrm{cm}^{2}$. This may place some constraints to models. In the QSO $1202+281$, for example, the covering factor would be nearly unity to produce the observed $H \beta$ flux if the density is $3 \times 10^{11} \mathrm{~cm}^{-3}$, and the size of the broad line region is 40 light-days (Gondhalekar, et al. 1986; Zheng, et al. 1987).

$L y \alpha$ still increases with density, leading to quite a high ratio of $L y \alpha / H \beta$ if the density is even higher. It is therefore suggested that a major part of the observed line emission is formed in a region of $10^{11} \mathrm{~cm}^{-3}$. Such an estimate is in general agreement with current observations. At even higher densities theoretical calculations are likely to produce a larger deviation from observation facts.

This work is supported in part by a NATO grant, 213-88. 


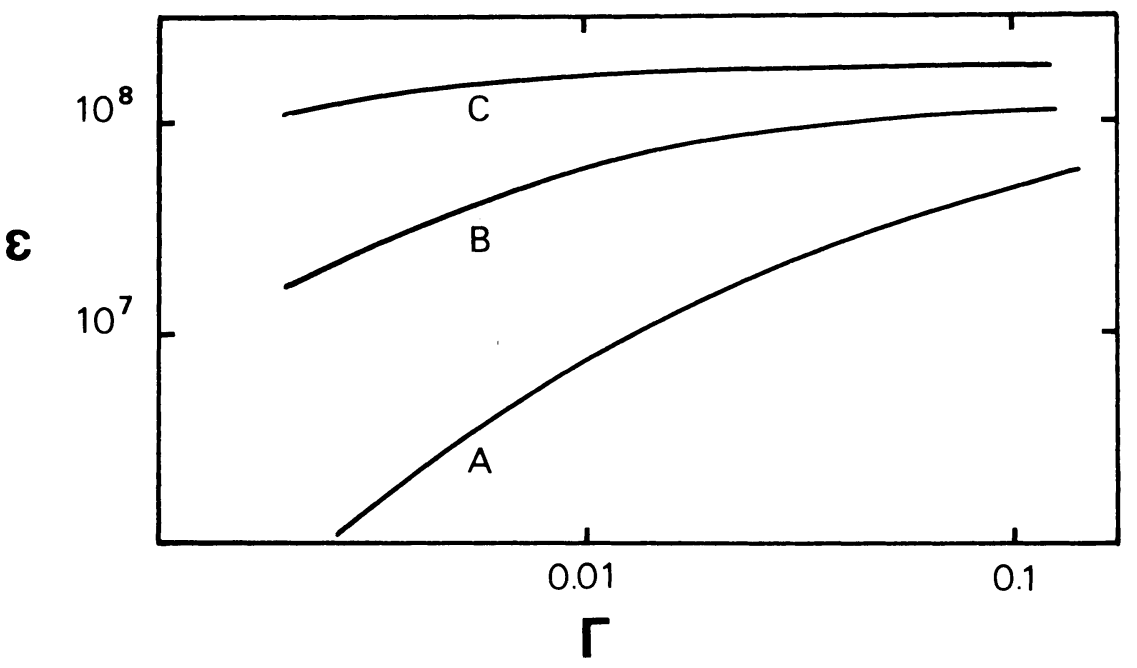

Figure 1. Total column emissivity, $\epsilon$, of $H \beta$, in units of $\mathrm{ergs} / \mathrm{s} / \mathrm{cm}^{2}$, vs. ionization parameter, $\Gamma$, for different densities: (A) $10^{10}$, (B) $10^{11}$, and (C) $10^{12}$, in units of $\mathrm{cm}^{-3}$ respectively.

\section{References}

Ferland, G. J., and Netzer, H. 1984, Publ. A.S.P., 96, 593.

Gondhalekar, P. M., O'Brien, P. T. and Wilson. R., 1986, in New Insights in Astrophysics, ESA SP-263, p. 711.

Peterson, B. M. 1988, Publ. A.S.P., 100, 18.

Zheng, W., Burbidge, E. M., Smith, H. E., Cohen, R. C., and Bradley, S. E. 1987, Ap. J., 322, 164.

Zheng, W., and Puetter, R. C. 1988, submitted. 